\title{
Magnetic field measurements during the ROSETTA flyby at asteroid (21)Lutetia
}

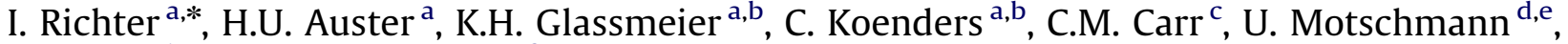 \\ J. Müller ${ }^{\mathrm{d}}$, S. McKenna-Lawlor ${ }^{\mathrm{f}}$ \\ a Institut für Geophysik und extraterrestrische Physik, Technische Universität Braunschweig, Mendelssohnstr. 3, 38106 Braunschweig, Germany \\ ${ }^{\mathrm{b}}$ Max Planck Institute for Solar System Research, Lindau, Germany \\ ${ }^{c}$ Imperial College London, London, UK \\ d Institut für theoretische Physik, Technische Universität Braunschweig, Mendelssohnstr. 3, 38106 Braunschweig, Germany \\ e DLR-Institut für Planetenforschung, Rutherfordstr. 2, 12489 Berlin, Germany \\ ${ }^{\mathrm{f}}$ Space Technology Ireland, National University of Ireland Maynooth, Co. Kildare, Ireland
}

\section{A R T I C L E I N F O}

Article history:

Received 13 May 2011

Received in revised form

18 August 2011

Accepted 19 August 2011

Keywords:

(21)Lutetia

Asteroids

ROSETTA

Magnetic field

Draping

Solar wind

\begin{abstract}
A B S T R A C T
On July 10, 2010, the ROSETTA spacecraft performed a flyby at asteroid (21)Lutetia at a solar distance of 2.72 AU. The spacecraft-asteroid distance at closest approach was $3120 \mathrm{~km}$. The magnetometers onboard ROSETTA were operating but did not detect any conclusive signature of the asteroid. Any magnetic field signature which could possibly be attributed to the asteroid was below $1 \mathrm{nT}$. Consequently an upper limit for the global magnetic properties of asteroid (21)Lutetia could be derived: maximum dipole moment $\leq 1.0 \times 10^{12} \mathrm{~A} \mathrm{~m}^{2}$, global maximum magnetization $\leq 2.1 \times 10^{-3} \mathrm{~A} / \mathrm{m}$, specific moment $\leq 5.9 \times 10^{-7} \mathrm{~A} \mathrm{~m}^{2} / \mathrm{kg}$. Draping of magnetic fields around the nucleus was sought, but evidence for this could not be clearly identified in the data. Plasma simulations taking into account the estimated upper limit of the magnetization and possible outgassing revealed interesting structures very close to the asteroid. The results obtained at Lutetia are contrasted with the results of other asteroid flyby results.
\end{abstract}

(c) 2011 Elsevier Ltd. All rights reserved.

\section{Introduction}

Asteroid research reveals essential facts about the evolution of our Solar System. The interaction of asteroids with the solar wind and the interplanetary plasma as well as information concerning asteroidal composition can be studied by investigating of the magnetic field in the vicinity of an asteroid. Different interaction scenarios are possible. If the asteroid consists of electrically conductive materials, a unipolar generator will be established and a current system (Ip and Herbert, 1982) that changes the topology of the external magnetic field can be induced. On the other hand, a purely magnetic interaction is possible if the asteroid contains remanent magnetized material. Such magnetization can be present if the parent body of the asteroid was initially magnetized by a dynamo (Weiss et al., 2008) and its field was frozen in during the cooling phase. Thus, asteroids with strong magnetic fields are believed to be fragments of larger parent bodies. Alternatively, the asteroid might have been

\footnotetext{
* Corresponding author.

E-mail address: i.richter@tu-bs.de (I. Richter).
}

subjected to one of a range of possible alteration processes (for example shocks, weathering or thermal influences), that, in the course of its lifetime, changed its magnetic properties (Weiss et al., 2010).

An opportunity to study the magnetization of an asteroid was provided by the flyby of asteroid (21)Lutetia (Barucci et al., 2007) by the ROSETTTA spacecraft. Lutetia is one of the largest known asteroids with a diameter of $\sim 100 \mathrm{~km}$.

\subsection{The ROSETTA project}

The ROSETTA project (Schwehm and Schulz, 1999; Glassmeier et al., 2007a) is a cornerstone mission of the European Space Agency (ESA) which was launched on March 2, 2004, from Kourou in French Guiana. Its main scientific objective is the investigation of comet 67P/Churyumov-Gerasimenko, which will be reached in 2014. In the course of its (10-year) journey through our Solar System, magnetic measurements were made during several swingbys of planet Earth (Glassmeier et al., 2007b; Eastwood et al., 2011) and of Mars (Edberg et al., 2008, 2009; Boesswetter et al., 2009) as well as during the flyby of asteroid (2867)Šteins (Auster et al., 2010). 


\subsection{The present study}

In this paper we report on magnetic field measurements made during the flyby of asteroid (21)Lutetia. The closest approach (C/A) of ROSETTA was at a distance of $3120 \mathrm{~km}$ from the asteroid's surface at 15:44:54 UTC on July 10,2010 . The relative flyby velocity was $15 \mathrm{~km} / \mathrm{s}$. ROSETTA approached the target from the upstream side above the ecliptic plane. At the time of the encounter (21)Lutetia was located at a distance of $2.72 \mathrm{AU}$ from the Sun and 3.05 AU from the Earth (Fig. 1). The approach profile of the flyby is displayed in Fig. 2.

In this paper we provide (Section 2) an account of the instruments used to obtain the magnetic measurements. An analysis of these measurements is contained in Section 3, where it is shown that, in addition to sensor effects which require correction, the magnetic field data are contaminated by spacecraft magnetic fields. These latter effects can be removed through calibration of the data, application of a long-term temperature model for the offset correction, and elimination of disturbances caused by ROSETTA's reaction wheels (RW). Further, the interference by several Lander instruments was identified.

Comparison of the data measured by the three onboard sensors ROMAP, RPC-MAG/OB and RPC-MAG/IB reveals (Section 4) upper limits for the magnetic properties of asteroid (21)Lutetia.

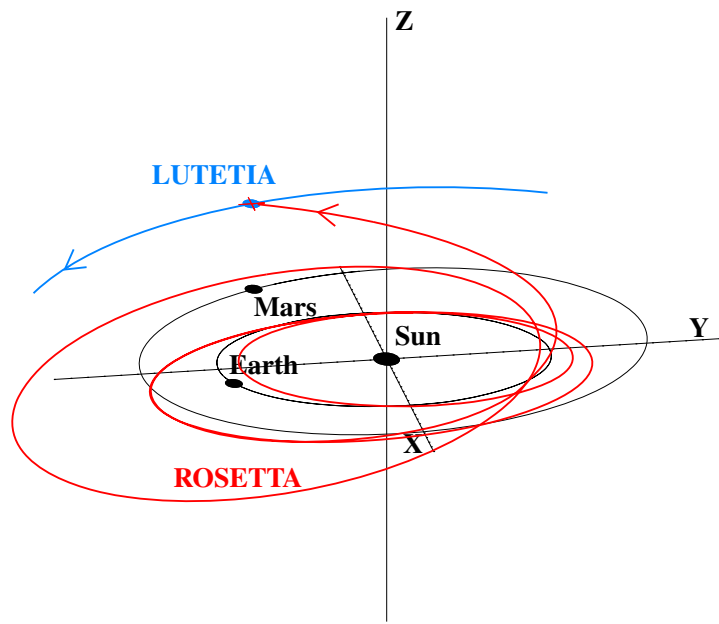

Fig. 1. The celestial situation at the flyby time of ROSETTA on July 10,2010 . Orbits are displayed in ECLIPJ2000 coordinates. Here $x$ points from the Sun to the vernal equinox, $y$ is in the ecliptic plane pointing against the orbital motion of the Earth and $z$ completes the right-handed coordinate system.

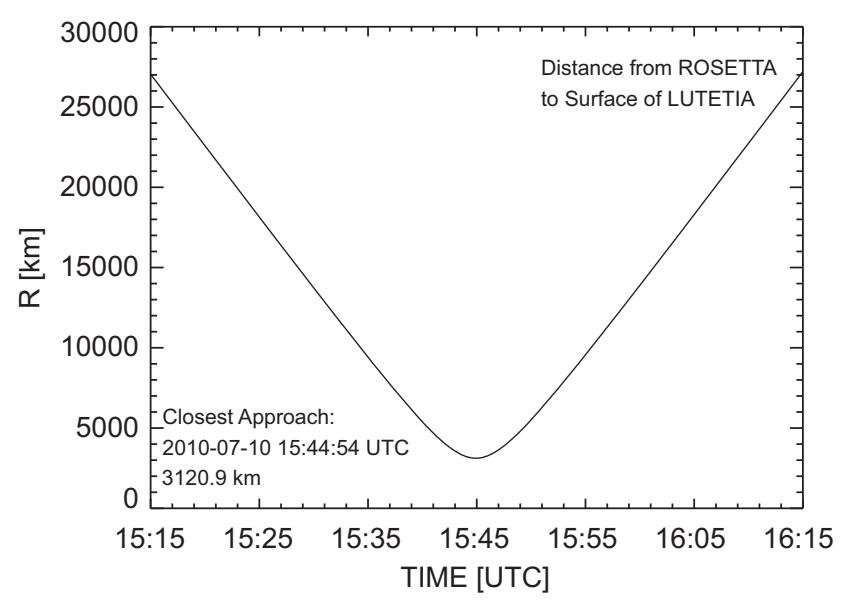

Fig. 2. Profile of the ROSETTA—Lutetia flyby distance during the encounter.
Also, using the calibrated data, the signature of asteroid (21)Lutetia was investigated in both the time and frequency domains.

Magnetic field draping effects measured at asteroid (951)Gaspra (Kivelson et al., 1993) are contrasted with ROSETTA measurements in Section 5 and various theoretically possible interaction scenarios are discussed in deriving upper limits for the magnetic properties of asteroid (21)Lutetia.

In Section 6, plasma simulations of structures close to Lutetia (taking into account possible outgassing and magnetization) are described. These would not have been seen at the C/A distance of $3120 \mathrm{~km}$. In Section 7 the flyby results are compared with data obtained during other asteroid encounters. Section 8 presents a general summary and conclusions.

\section{The magnetometers}

The ROSETTA spacecraft is equipped with two independent magnetometer systems: namely the ROSETTA Lander magnetometer ROMAP (Auster et al., 2007) and the orbiter magnetometer RPC-MAG (Glassmeier et al., 2007b), the latter of which is part of the ROSETTA plasma consortium RPC (Carr et al., 2007). Both instruments (which were developed at the Institute for Geophysics and Extraterrestrial Physics in Braunschweig) are 3-axis fluxgate magnetometers (FGM) with a resolution of $0.03 \mathrm{nT}$. RPC-MAG can operate within the measurement range of $\pm 16000 \mathrm{nT}$, and over a wide temperature range of $-150^{\circ} \mathrm{C}$ to $+150{ }^{\circ} \mathrm{C}$. In the normal operating mode the instrument is run at a $1 \mathrm{~Hz}$ sampling rate. In burst mode the vectors are sampled at $20 \mathrm{~Hz}$. A prototype of the RPC-MAG instrument has already been flown successfully onboard NASA's DEEP SPACE 1 mission where it detected the magnetic signature of asteroid (9969)Braille (Richter et al., 2001) and characterized the magnetic properties of comet 19P/Borrelly (Richter et al., 2011).

The two RPC orbiter FGMs (inboard: IB, outboard: OB) are located outside the spacecraft on a deployed, $1.55 \mathrm{~m}$-long boom. The separation distance between the sensors is $15 \mathrm{~cm}$. The Lander magnetometer ROMAP is located on a stowed boom inside the Lander PHILAE (this boom will be deployed at the target comet 67P/Churyumov-Gerasimenko).

During the asteroid (21)Lutetia flyby, the magnetometers were switched on from July 7, to July 13, 2010.

\section{Data processing}

\subsection{Standard calibration}

As a first step in data processing, the raw data obtained are adjusted using the results of previous ground calibrations, namely by the application of the temperature-dependent sensitivity and misalignment matrices. The temperature dependence of the sensor offsets was eliminated through the utilization of a thirdorder-polynomial temperature model. This latter model was developed using magnetic field measurements made during quiet phases over a 6 years period of cruise. The temperature dependencies in the range of $-150{ }^{\circ} \mathrm{C}$ to $+50{ }^{\circ} \mathrm{C}$ were specified individually for each single sensor component. The obtained calibrated data are rotated into s/c-coordinates to establish a common coordinate system.

\subsection{Disturbance analysis}

Fig. 3 exhibits calibrated data of the RPC-MAG/OB, the RPCMAG/IB and the ROMAP magnetometer for July 10, 2010 in spacecraft-coordinates. Closest approach occurred at 15:44:54 


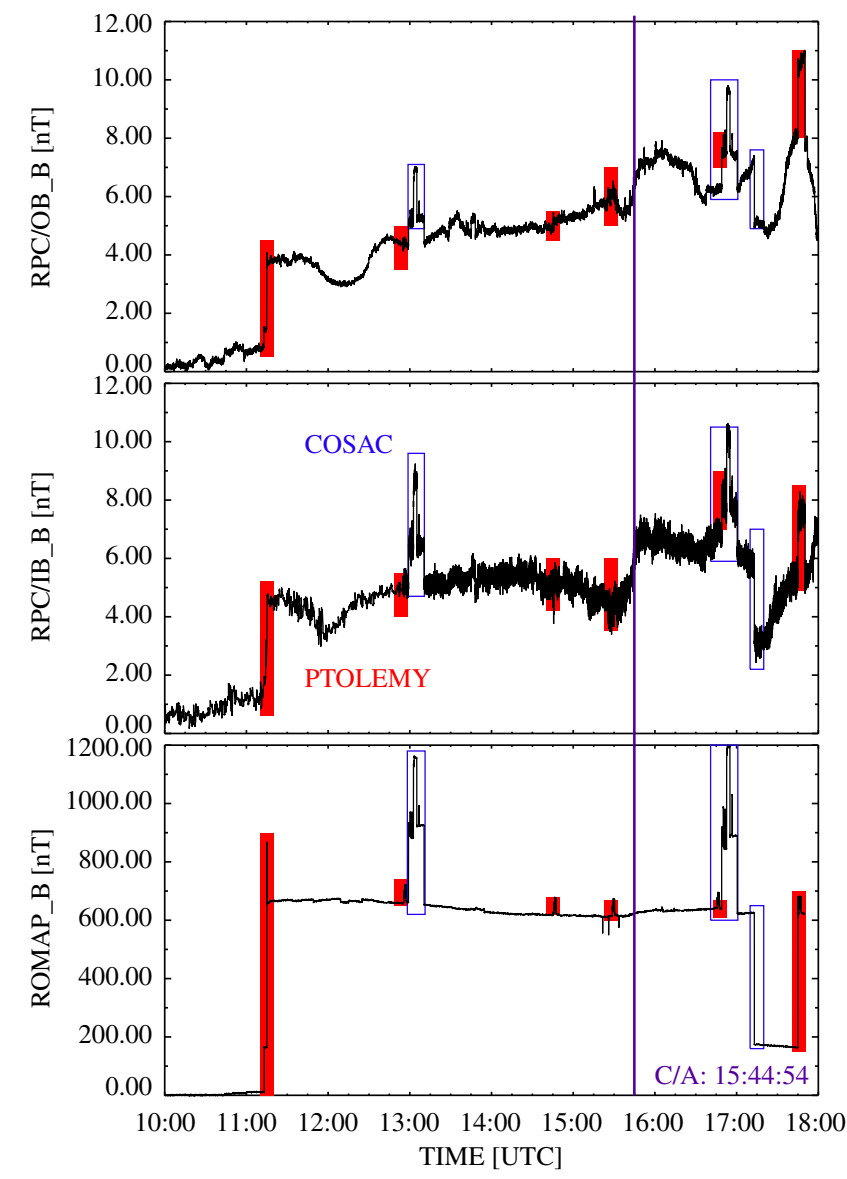

Fig. 3. This figure shows the magnetic field magnitude measured by the RPCMAG/OB, the RPC-MAG/IB and the ROMAP magnetometer. The data of an $8 \mathrm{~h}$ interval around $C / A$ are displayed in spacecraft-coordinates. Disturbances produced by the PTOLEMY (red) and COSAC (blue) instruments are marked. Note the 10 times larger scale of the ROMAP data.

UTC. As the sensors are mounted at short distances from the spacecraft/Lander, the FGM observations are strongly influenced by various disturbances produced by other payload instruments and by spacecraft-subsystems. Thus, the most prominent feature of the shown time series is not an asteroid signature but the disturbances produced by the Lander instruments PTOLEMY (Wright et al., 2007) and COSAC (Goesmann et al., 2007).

The different strengths of the disturbances seen on ROMAP and RPC-MAG are worth mentioning. If PTOLEMY is powered on or off a magnetic field jump of about $600 \mathrm{nT}$ is seen on ROMAP, whereas the response on RPC-MAG is only $\sim 4 \mathrm{nT}$ due to its greater distance from the disturbance source. If PTOLEMY is operating in its so called sniff-mode, RPC-MAG is disturbed in the order of $1 \mathrm{nT}$. The COSAC instrument was found to interfere, generating signatures of about $600 \mathrm{nT}$ at the location of ROMAP and about $4 \mathrm{nT}$ at RPC-MAG. PTOLEMY and COSAC were not operating continuously around C/A and therefore, the quality of the magnetic field measurements obtained at that specific time was not degraded by the above mentioned instruments.

Comparison of the RPC-MAG/OB and IB data shows also that the spacecraft-noise registered by the latter sensor is about three times higher than the noise measured by the OB sensor. This behavior is explained by the different mounting positions of the different sensors with respect to the spacecraft-body. Although their separation is only $15 \mathrm{~cm}$ the influence of the spacecraft on the OB sensor was significantly less.
Further disturbances are revealed on transforming the measured data into the frequency domain. Fig. 4 shows the dynamic spectrum of the magnetic power spectral density (color coded) measured by the $\mathrm{OB}$ sensor in a 2 hour interval around $\mathrm{C} / \mathrm{A}$ (abscissa). As the data were sampled at 20 vectors per second, a spectrum can be calculated up to the Nyquist frequency of $10 \mathrm{~Hz}$ (ordinate). The horizontal lines at about $3.2 \mathrm{~Hz}$ were caused by interference from the RPC-LAP instrument (Eriksson et al., 2007). The funnel shaped signature centered around C/A was generated by ROSETTAs rotating reaction wheels (RW). These four wheels were spinning with varying, different frequencies to maintain a controllable spacecraft attitude. Therefore, a wide frequency band was observed around C/A as the spacecraft had to stay nadirpointed during the close flyby. The original RW frequencies were well above the Nyquist frequency of MAG sampling but appeared as aliased frequencies. Both the RPC-LAP disturbance and the RW impact can be automatically eliminated using calibration software.

\subsection{The asteroid flyby rehearsal}

On March 14/15, 2010, a Lutetia flyby rehearsal was executed to test and validate the procedures for the real flyby in July, 2010. Some payload instruments (including the magnetometers) were switched on during this campaign to realize the chance to obtain a reference measurement in the absence of a real asteroid. With the knowledge provided by these reference data it is possible to more correctly interpret the flyby data.

An increased level of spectral activity level was observed exactly at $\mathrm{C} / \mathrm{A}$ in the low frequency band up to $\sim 400 \mathrm{mHz}$ (Fig. 4). It is tempting to interpret this pattern as an asteroid-related signature.

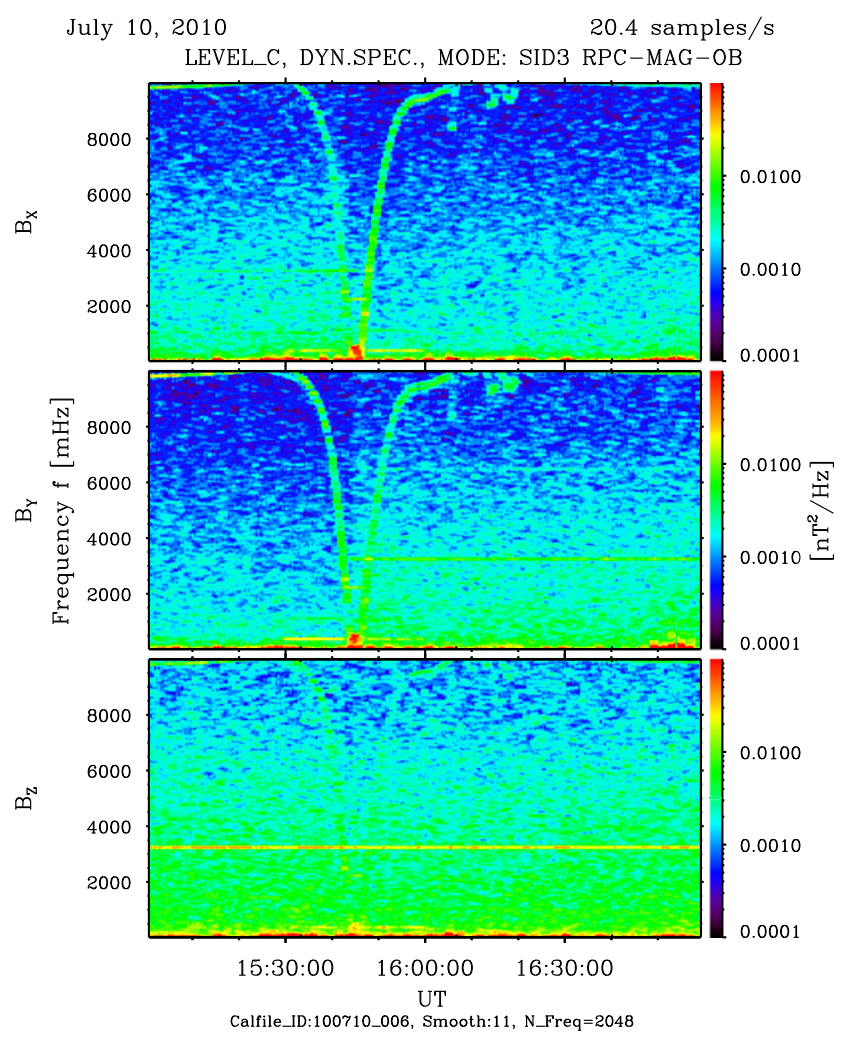

Fig. 4. The dynamic spectrum of the magnetic field during the asteroid (21)Lutetia flyby measured by RPC-MAG. The plot shows the three components of the power spectral density versus time. Details of the visible spectral lines are discussed in the main text. 
However, this signature is also present in the rehearsal data (not shown here), so that the source must have been located aboard the spacecraft and not at asteroid (21)Lutetia.

\subsection{The HAFv.2 model of the interplanetary field}

For a proper determination of the magnetic field DC-level the knowledge of the actual interplanetary field (IMF) is helpful. There was no possibility to use the IMF data measured by, e.g., WIND or ACE, as these spacecrafts were not located at a conjugated field line with respect to the position of ROSETTA at asteroid (21)Lutetia. Therefore, we draw upon the HakamadaAkasofu-Fry-version 2 (HAFv.2) model, which provides predictions of solar wind and magnetic field parameters under both "quiet" and "event driven" conditions out to several AU in the heliosphere (Fry et al., 2003; McKenna-Lawlor et al., 2008).

This kinematic model ingests radial solar wind speed and radial IMF at $5 R_{s}$ (solar radii). For July 10,2010 , this information was obtained from SCSS (source current surface sheet) maps available from the NOAA Space Weather Prediction Centre using a procedure developed by Wang and Sheely (1990) and extended by Arge and Pizzo (2000), which is known by the acronym WSA (Wang-Sheely-Arge). The WSA algorithm uses daily magnetograms from Mount Wilson Solar Observatory on a $5^{\circ} \times 5^{\circ}$ grid in heliolatitude and heliolongitude to provide radial values of the solar wind speed and field strength at $5 R_{S}$ (the inner boundary of the model). These values are input to the HAFv.2 code, modeling the non-uniform, time-dependent, solar wind conditions that flow outward through the heliosphere from $5 R_{s}$, initialized by the (daily) synoptic line of sight magnetograms.

On the occasion of the Lutetia encounter, the model indicates that ROSETTA was located in an 'away sector' (IMF lines directed outward from the Sun) under what (within the time resolution of the WSA) were quiet solar wind conditions.

\subsection{Determining the absolute DC level of the external magnetic field}

The next step in processing the raw data involves determining the absolute DC level of the external magnetic field.

It is worthwhile to point out that it was not the sensor offsets of the magnetometers but rather the changing spacecraft-residual fields-due to high payload and spacecraft-activity, that gave rise to difficulties in adjusting the zero level correctly. The sensor offsets were already known within the range up to $1 \mathrm{nT}$ from the ground calibration and by application of the, already mentioned, long term temperature model.

The absolute magnetic field value cannot be determined directly as the in-flight calibration technique for spinning satellites (e.g. Anderson et al., 2001) is not applicable for ROSETTA. Also, the standard calibration method in the solar wind (Hedgecock, 1975; Leinweber et al., 2008) cannot be utilized due to the mentioned disturbances.

One reasonable way to estimate the DC-level is to assume a Parker-type solar wind magnetic field in the assigned region. Then to set the mean residual field components measured by the sensors in a quiet period before the encounter to the theoretically expected values of the Parker field at that location at that time. The Parker field can be calculated by means of the spatial Parker field decay law (e.g. Musmann et al., 1977). This approach is reasonable under quiet conditions, as forecasted by the above presented HAFv2.0 model.

For the avoidance of these general models, however, we finally adjusted the data just to zero at the start of the measurements and fine tuned the offsets using the rotation angles of the spacecraft flip which occurred in the time interval $C / A \pm 15 \mathrm{~min}$. The spacecraft $y$-axis was kept stable in space, whereas the angle between the spacecraft $x$-axis an the Sun-direction changed by $\sim \pm 90^{\circ}$. Also the angle between the spacecraft $z$-axis and the Sun-direction changed by $\sim 160^{\circ}$. This was definitely not a full rotation around three axes but could nevertheless be used to minimize the effect of incorrect zero-levels which would emerge during the transition from spacecraft-coordinates to a fixed celestial coordinate system.

The remaining small uncertainties in the magnetic field zero level (originating either from spacecraft-generated disturbances or from sensor offsets) do not affect our final conclusions, as we are only interested in relative changes caused by the asteroid.

\subsection{The CSEQ coordinate system}

In the last analysis step the data are averaged to $1 \mathrm{~s}$ means and, taking into account the spacecraft-attitude information (provided by SPICE kernels, Acton, 1996), the magnetic field data are available in asteroid centered solar equatorial coordinates (CSEQ), where $x$ points towards the Sun, $z$ is the component of the rotation axis of the Sun which is perpendicular to $x$, and $y$ completes the right-handed system ( $y$ is parallel to the Sun's equatorial plane). This coordinate system is the one chosen for the ongoing analysis, because the $x$-axis as the asteroid-Sun line is the major symmetry axis and the Sun equatorial plane is the major symmetry plane for the solar wind.

Using the above described spacecraft rotations an average solar wind magnetic field during the flyby of $B_{\text {ext }} \sim[0,-1.4,1.1] \mathrm{nT}$ (CESQ) was calculated. Its magnitude of $1.8 \mathrm{nT}$ is close to the mean theoretical Parker field value of $1.9 \mathrm{nT}$ at this location.

\section{The magnetic signature of asteroid (21)Lutetia}

Fig. 5 displays $1 \mathrm{~s}$ means of the magnetic field components and the total magnitudes measured by all three instruments in CSEQcoordinates. The time interval shown covers $\pm 15 \mathrm{~min}$ around $\mathrm{C} / \mathrm{A}$ at 15:44:54 UT. The RPC-MAG/OB (red) and RPC-MAG/IB (green) data are scaled to a span of $3 \mathrm{nT}$ (left scale). The ROMAP data (blue) are displayed on a 10 times broader scale (right scale; 30 nT span).

The data of both the RPC-MAG sensors appear similar and show variations of less than $2 \mathrm{nT}$ within the exhibited time interval. Both time series match reasonably (which reflects a proper calibration performance). The spacecraft-noise measured by the IB sensor is about 2-3 times higher than that of the OB, because the IB sensor is mounted $15 \mathrm{~cm}$ closer to the spacecraftbody with all its disturbance sources.

The ROMAP data show much larger variations and also a different long term behavior in the considered time interval. These different behaviors provide a further strong indication of the presence of spacecraft-generated disturbances.

A detailed inspection of the RPC-MAG/OB components reveals sharp field changes of about $0.5 \mathrm{nT}$ at $\sim \mathrm{C} / \mathrm{A}-1 \mathrm{~min}$ and an increased level of fluctuations until $\sim \mathrm{C} / \mathrm{A}+2 \mathrm{~min}$. The $\mathrm{RPC}-$ MAG/IB data show, in principal, similar behavior, but the signature is overlaid by increased noise.

The above feature, however, looks promising and might possibly be attributed to the influence of asteroid (21)Lutetia. To test this hypothesis, the complementary ROMAP data were inspected. The latter data show, in addition to various disturbances caused by different switched sources on the spacecraft, a similar jump at the considered time. However, the peak-to-peak jump height measured by ROMAP is $\sim 7 \mathrm{nT}$, and this jump seems to be the consequence of an event (an unknown, spacecraftrelated phenomenon) that happened half a minute before. 
This obvious difference in the data measured by RPC-MAG shows unambiguously that this effect was caused by the spacecraft and not by the asteroid (because the influence of an external field source would appear the same at all sensors (far field), whereas sources located on the spacecraft generate different signatures at different distances).

Besides the comparison with ROMAP data the Lutetia flyby rehearsal data set, already mentioned above but not shown, were closely investigated. These rehearsal data also exhibited an increased turbulence level for $3 \mathrm{~min}$, starting at the simulated C/A-1 min. Although there is a data gap of $30 \mathrm{~s}$ in the rehearsal data directly at the simulated C/A, these reference data are valuable for making a proper assessment of the real flyby data and provide a second proof that no magnetic signature caused by asteroid (21)Lutetia was detected.

Possibly there is a hidden structure (much less than $1 \mathrm{nT}$ ) in the flyby data caused by the asteroid but if so this is masked by spacecraft-fields and cannot be isolated. Therefore, $\sim 1 \mathrm{nT}$ provides an upper limit for detecting the influence of asteroid (21)Lutetia at the C/A distance of $3120 \mathrm{~km}$.

For future analysis the knowledge of relevant plasma parameters is desirable. Although ROSETTA is itself equipped with a full plasma package (RPC), auxiliary data are not available for the flyby. RPC-ICA (Nilsson et al., 2007) suffered a switch off due to thermal reasons; the field of view of RPC-IES (Burch et al., 2007) pointed in an unfavorable direction; RPC-MIP (Trotignon et al., 2007) is not designed for low activities at asteroids, and RPC-LAP (Eriksson et al., 2007) did not detect an asteroidal signature.

\section{Investigation of magnetic field draping}

In what follows, the pertaining magnetic field directionality is examined to investigate the possibility of field draping around asteroid (21)Lutetia. These data are compared with the field draping observed during the Galileo flyby of asteroid (951)Gaspra in 1991. The latter flyby data (Kivelson et al., 1995) are shown in Fig. 6. In the upper panel the flyby trajectory and the projection of the magnetic field in the $x y$-plane are displayed in CSEQ-coordinates. Inbound and outbound the magnetic field direction is similar, whereas in an interval of about $4 \mathrm{~min}$ around $\mathrm{C} / \mathrm{A}$ at about 22:36:40 the field significantly turned due to an interaction with the asteroid. Kivelson et al. (1993) interpreted this signature to represent a disturbed field inside the asteroidal magnetosphere. The draping angle is shown in the second panel. It can clearly be seen that the crossing of the assumed magnetospheric boundaries caused the angle to change by $\sim 40^{\circ}$ relative to the undisturbed solar wind direction. The theoretically expected angle of the Parker spiral at the location of Gaspra (at 2.2AU distance from the Sun) is indicated by a green line.

In ROSETTA/Lutetia data displayed in Fig. 7, the upper panel shows the flyby trajectory in the $x y$-plane. The projected magnetic field direction in the $x y$-plane, calculated from the RPC-MAG/OB data, is plotted along the trajectory. These data look similar during both the inbound and outbound phases. About $5 \mathrm{~min}$ before $C / A$, however, the field starts to rotate uniformly until about $\mathrm{C} / \mathrm{A}+2 \mathrm{~min}$ when the projected angle jumped back abruptly. To provide a more quantitative analysis the angle between the magnetic field and the asteroid-Sun direction is plotted (black curve in the bottom panel). The temporal behavior is characterized by a steady increase in the draping angle until about C/A-1 min, starting at $\sim 50^{\circ}$. Then the values decrease smoothly to $\sim 85^{\circ}$ in the outbound leg. Although small variations in the angle can be seen, the behavior is completely different to what was found in the case of Gaspra, where the angle jumped back and forth by about $40^{\circ}$. The theoretical expected angle of the
Magnetic field data: ROSETTA @ (21)

Lutetia, CSEQ-Coordinates

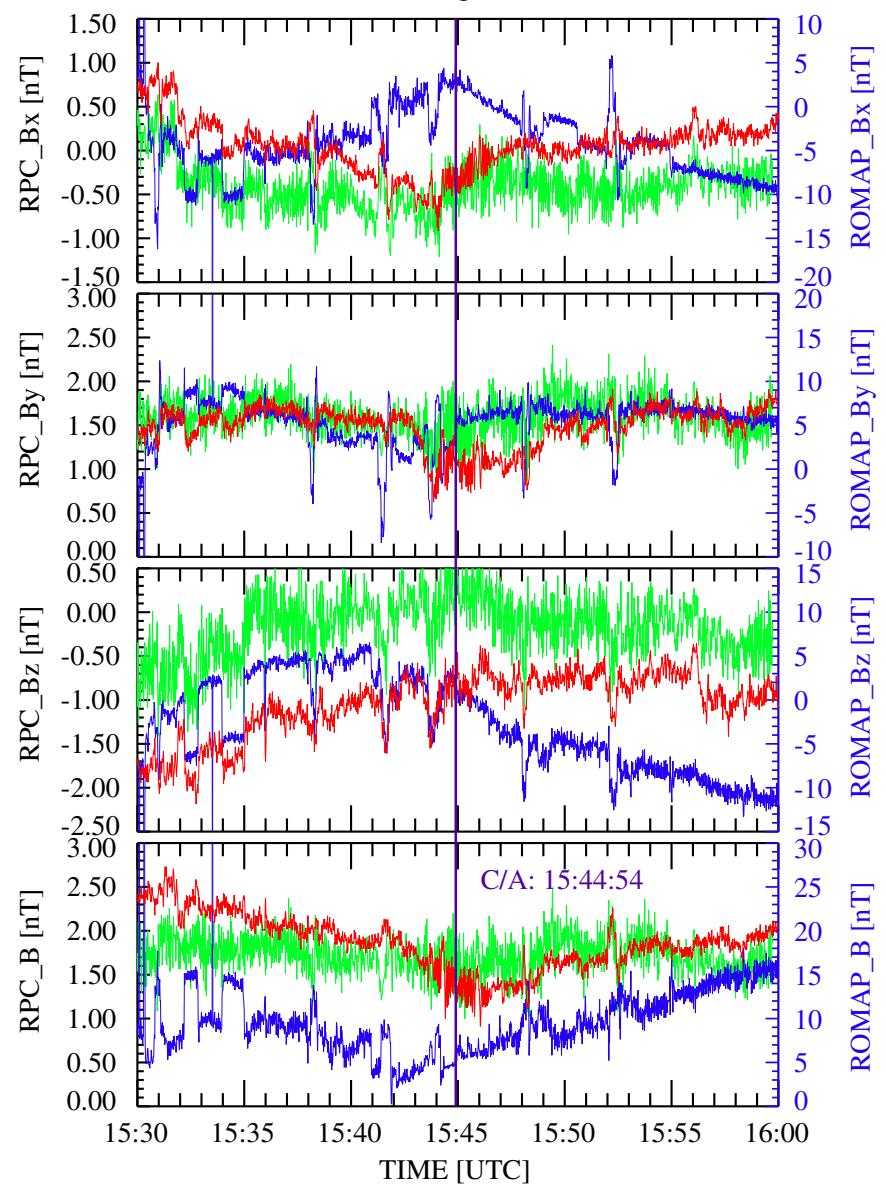

Fig. 5. This figure shows the magnetic field time series of the OB (red), IB (green), and ROMAP (blue) magnetometers measured during a $30 \mathrm{~min}$ interval around C/A (vertical line) in CSEQ-coordinates ( $x$ is pointing from the asteroid to the Sun, $z$ is the component of the rotation axis of the Sun which is perpendicular to $x$, and $y$ completes the right-handed system). Note the different scales for OB,IB (left side) and ROMAP (right side). The ROMAP-scale is 10 times that of the RPC-scale.

Parker spiral ( $70^{\circ}$ at $2.72 \mathrm{AU}$ from the Sun $)$ is indicated by a green line. At both Lutetia and Gaspra the observed magnetic field angles were, during most of the time, greater than the Parker angle.

To investigate the draping angle, the uncertainties in the absolute value of the field components have to be considered and the error in the calculated angles taken into account. The measured field can be expressed as the sum of the time dependent external interplanetary field $B_{\text {ext }}(t)$ and a fixed but unknown disturbance field $B_{\text {err }}$ (spacecraft-residual field and sensor offset). ${ }^{1}$ The complete field is then given by $B_{i}(t)=B_{\text {ext, } i}(t)+B_{\text {err, }}$ with $i=\{x, y, z\}$. The required magnetic field draping angle $\alpha$ can then be calculated as $\alpha(t)=\arctan \left(B_{y}(t) / B_{x}(t)\right)$. The error in this angle, caused by uncertainty in the sensor offset and in the spacecraft field, is estimated as $\Delta \alpha=\sqrt{\left(\partial \alpha / \partial B_{\mathrm{err}, x} \cdot \Delta B_{\mathrm{err}, x}\right)^{2}+\left(\partial \alpha / \partial B_{\mathrm{err}, y} \cdot \Delta B_{\mathrm{err}, y}\right)^{2}}$.

Under the assumption of offset/disturbing field errors of the maximum $\Delta B_{\text {err }}=0.5 \mathrm{nT}$, the error in the draping angle is

\footnotetext{
1 This assumption is made although the disturbance is obviously changing with time. For the short interval of interest, the frequent changes in the disturbances are neglected since, for the draping angle error assessment, only the coarse trend of the disturbance is significant.
} 

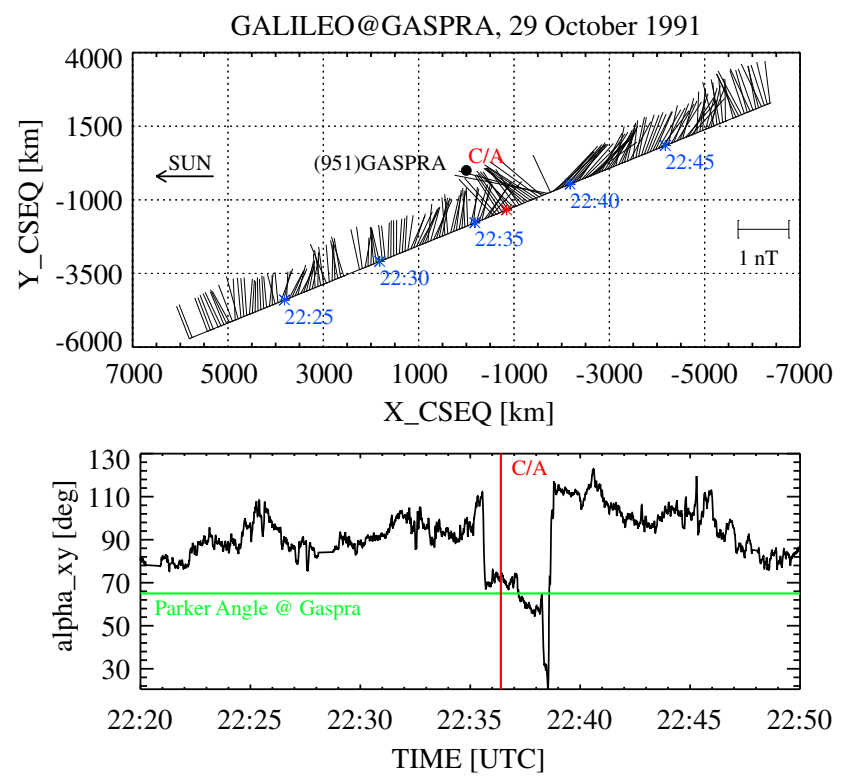

Fig. 6. Magnetic field draping at asteroid (951)Gaspra measured during the Galileo flyby in 1991. The upper panel shows the $x y$-plane projection of the magnetic field along the Galileo-trajectory in Gaspra-centered-solarequatorial-coordinates. The second graph exhibits the projected angle of the magnetic field.
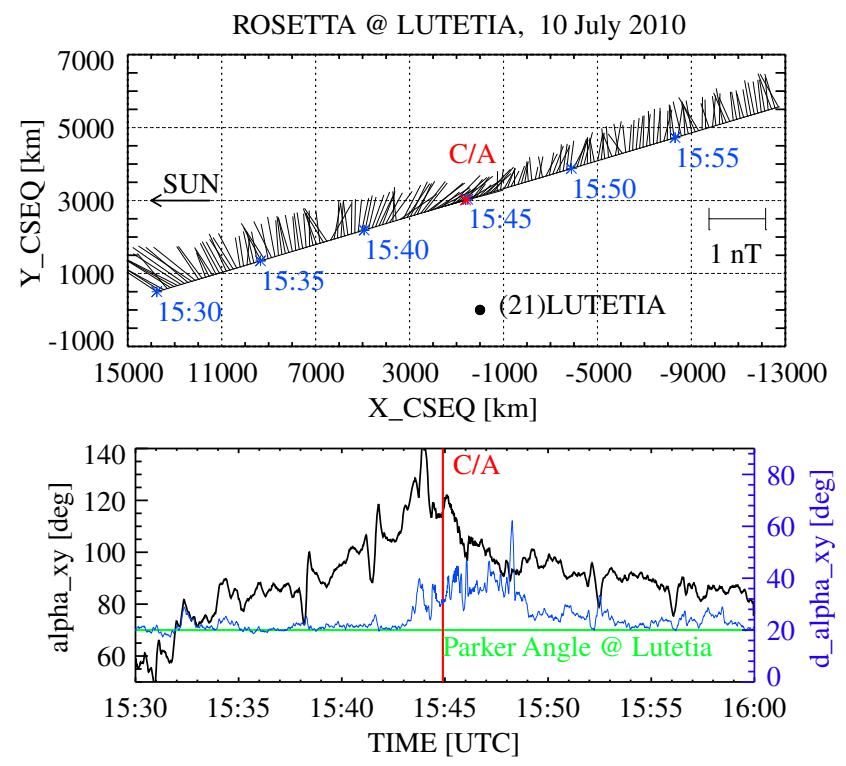

Fig. 7. Magnetic field draping at asteroid (21)Lutetia measured during the ROSETTA flyby. The upper panel shows the $x y$-plane projection of the magnetic field along the ROSETTA-trajectory in Lutetia-centered-solar-equatorial (CSEQ)coordinates. The second graph exhibits the projected angle (black) of the magnetic field. The blue line (right scale!) shows an estimate of the expected error in the angle. The theoretical Parker angle is shown as green line. Details are discussed in the text.

represented by a blue curve in the bottom panel of Fig. 7. The errors during the inbound and outbound phases are relatively constant at $\sim 25^{\circ}$. Near $\mathrm{C} / \mathrm{A}$, however, the error increases to values of $\sim 40^{\circ}$. A more conservative assumption for $\Delta B_{\text {err }}=1 \mathrm{nT}$ would cause an angle error of $\sim 40^{\circ}$ in the far approach phases and $\sim 70^{\circ}$ near C/A. The errors are as high as the disturbance level and the offsets are of the order of the measured field. Taking all these estimates into account, we conclude that the possible existence of very faint draping around asteroid (21)Lutetia, which is much less pronounced than at asteroid (951)Gaspra, cannot be unambiguously demonstrated. Due to the large flyby distance, involved, extensive spacecraft disturbances and the very small solar wind/asteroid signal, the draping angle uncertainties are so large that a definite statement cannot be made.

\section{Theoretical considerations}

In this section a theoretical analysis is presented to throw light on the measured results. Different scenarios are considered in order to provide a quantitative assessment of potentially observable signatures, acquired under different model circumstances.

\subsection{Description of Lutetia as a magnetic dipole}

Assumption: Asteroid (21)Lutetia can be described by a simple dipole characterized by $r^{-3}$ decay

$B_{\mathrm{c} / \mathrm{a}}\left(r_{\text {body }}, r_{\mathrm{c} / \mathrm{a}}\right)=\mu_{0} \frac{M}{3}\left(\frac{r_{\text {body }}}{r_{\mathrm{c} / \mathrm{a}}}\right)^{3}$

with magnetization $M \sim 100 \mathrm{~A} \mathrm{~m}^{2}$, which is of the order of the global magnetization of asteroid (9969)Braille (Richter et al., 2001) and of (951)Gaspra (Kivelson et al., 1993). Under this premise an asteroid with radius $r_{\text {body }}=50 \mathrm{~km}$ would cause only a field change of the order of $B_{\mathrm{c} / \mathrm{a}}=0.2 \mathrm{nT}$ at a flyby distance of $r_{\mathrm{c} / \mathrm{a}}=3000 \mathrm{~km}$.

Already from this coarse estimate it is clearly evident that, even under the assumption of relatively large magnetization, the direct detection of any dipole is unlikely in the noisy environment of ROSETTA.

\subsection{Application of Greenstadt's constraints to Lutetia}

As a second scenario we suppose the asteroid to act as an obstacle for the streaming solar wind plasma, thereby building up a mini-magnetosphere. This can be achieved if the body is sufficiently magnetized or electrically conductive. Three quantitative constraints for the existence of such a magnetosphere were formulated by Greenstadt (1971). The application of his formulae to asteroid (21)Lutetia led (1) to a minimum required surface field $B_{\mathrm{s}}=31 \mathrm{nT}$ for balancing the solar wind pressure at the stopping distance (2) of $r_{\mathrm{s}}=3.1 \mathrm{~km}$. The used stopping distance $r_{s}=\sqrt{R_{p} R_{e}}$, which is defined as a mean of proton and electron gyro radii, is the least distance from the body at which the stopping field $B_{\mathrm{s}}$ can inhibit the solar wind protons from hitting the surface.

To guarantee the lateral stability of the asteroid structure and get rid of edge effects, the third condition (3) must be met: i.e. the radius of curvature of the field of the asteroid must exceed the proton gyro radius $R_{p}$ in the stopping field $B_{s}$. This requires a stopping field greater than $\sim 5200 \mathrm{nT}$ corresponding to a minimum dipole moment of $3 \times 10^{15} \mathrm{~A} \mathrm{~m}^{2}$, with a minimum magnetization of $6.2 \mathrm{~A} / \mathrm{m}$ and a minimum specific moment of $1.8 \times 10^{-3} \mathrm{~A} \mathrm{~m}^{2} / \mathrm{kg}$. The subsolar distance of the magnetopause in this model would be $r_{\mathrm{mp}} \sim 270 \mathrm{~km}$ with a derived minimal magnetospheric diameter of $\sim 810 \mathrm{~km}$ incorporating a flaring factor of 1.5 (Wallis, 1986). This implies that even if a magnetosphere were present, its existence would not have been detected by ROSETTA as the flyby distance was about four times too large.

\subsection{Comparison between Lutetia and lunar measurements}

Measurements at the Moon were used for comparison with those at Lutetia. Ness (1971) quoted an upper limit for the lunar magnetic moment in the order of $10^{16} \mathrm{~A} \mathrm{~m}^{2}$ or less. A lunar bow 
shock does not exist but the generation of Mach cones and solar wind perturbations in the wake of the Moon were observed.

Similar structures were not detected at Lutetia as the spacecraft trajectory did not cross the umbra while also the dipole moment seems to be much lower than the lunar moment (see below).

\subsection{Prediction of standing whistler waves}

Linear 3D-simulations of a magnetized asteroid (Baumgärtel et al., 1997) revealed that an asteroid with a magnetic moment of $\geq 10^{12} \mathrm{~A} \mathrm{~m}^{2}$ can generate observable magnetic fluctuations $(\delta B / B \geq 0.1)$ at a distance of $\sim 1000 \mathrm{~km}$. These disturbances should be visible as a pattern of standing whistler waves but could not be detected during the Lutetia flyby, leading to the conclusion that the dipole moment of this asteroid must be less than $10^{12} \mathrm{~A} \mathrm{~m}^{2}$.

\subsection{Excursus: Runcorn's sphere}

A general remark regarding asteroidal magnetization has to be made at this point. Runcorn (1975a,b) showed impressively that an ideal sphere with an internally generated magnetic field (of any topology) yields a magnetization in the spherical shell that does not produce any magnetic field at the exterior of this body. If therefore, at any time in the past an internal source (e.g. a dynamo) caused a magnetization inside the asteroid, this magnetization could remain after the internal source vanishedbut a magnetic field outside the body would not exist. However, the absence of a magnetic signature measured during a flyby does not necessarily prove that a celestial body is not magnetized.

\subsection{Hybrid simulation of an asteroid with outgassing and magnetization}

Measurements with ROSETTAs Spectrometer for Ion and Neutral Analysis - ROSINA - (Balsiger et al., 2007) revealed an upper limit for Lutetia's water production rate. Altwegg et al. (this issue) calculated the water density at the surface of the asteroid by application of an exospheric model to the measured water density. Taking into account a simplified temperature model and considering exospheric losses by Jeans escape and photo ionization an upper limit for the outgassing rate of the order of $Q_{\mathrm{H}_{2} \mathrm{O}} \sim 3.2 \times 10^{25} \mathrm{~s}^{-1}$ could finally be estimated. This limit is remarkably high and matches a weak comet better than an asteroid.

To investigate the influence of asteroid outgassing combined with its magnetic moment on the plasma environment, we performed a 3D hybrid simulation using the simulation code A.I.K.E.F. (Adaptive Ion-Kinetic Electron-Fluid). For a detailed description of this code see Müller et al. (2011). A former version of the code was used by Bagdonat and Motschmann (2002) to examine the interaction of a weak comet with the solar wind. Also applying the same code, Simon et al. (2006) investigated the interaction between the solar wind and an asteroid with strong magnetization but without outgassing. The A.I.K.E.F code is based on a hybrid model which describes the ions as kinetic particles and the electrons as a massless fluid.

However, the electric and magnetic fields are calculated at the nodes of an hierarchal mesh. The highest resolution of $\approx 16 \mathrm{~km}$ per gridcell can be found around the asteroid deemed to be placed in the middle of the box. We modeled this asteroid as a sphere with a radius of $50 \mathrm{~km}$.

Every ion that enters the sphere is deleted. The electrical conductivity of the asteroid is set at $10^{-5} \mathrm{~S} / \mathrm{m}$ and the inferred magnetic moment of $10^{12} \mathrm{~A} \mathrm{~m}^{2}$ is used to represent a dipole magnetic field oriented in the $z$-direction. The outgassing is assumed to be like that of a weak comet and the density growth rate of the water ions produced is given by $\partial n_{h i}(r) / \partial t=$ $v Q /\left(4 \pi u_{g} r^{2}\right) \exp \left(-v r / u_{g}\right)$, where $r$ is the radial distance to the center of the asteroid, $u_{g}=1 \mathrm{~km} / \mathrm{s}$ is the radial neutral velocity and $Q$ is the gas production rate. We used the measured value from Altwegg et al. (this issue) with $Q=3.2 \times 10^{25} \mathrm{~s}^{-1}$. At 2.7 AU the photoionization frequency is about $v=1.3 \times 10^{-7} \mathrm{~s}^{-1}$ (Hansen et al., 2007).

The box has a length of $6000 \mathrm{~km}$ in every direction. The undisturbed solar wind moves parallel to the $x$-axis with a velocity of $400 \mathrm{~km} / \mathrm{s}$. We used a solar wind background density of $n_{0}=1.4 \mathrm{~cm}^{-3}$. The magnetic field has a magnitude of $1.8 \mathrm{nT}$, it is in the $x y$-plane and the angle between the solar wind velocity and the magnetic field is $70^{\circ}$, which matches the Parker angle at this heliocentric distance.

The results of the hybrid simulations are shown in Fig. 8. It is known from weak comets (see Bagdonat and Motschmann, 2002) that the water ions are picked up by the solar wind. This leads to cycloidal motion of the water ions and the development of a tail. The beginning of this water ion tail can be seen in Fig. 8(d), which displays the water ion density. Another signature of this tail can be found in the magnetic field strength in Fig. 8(a).

Another feature in the plasma environment of the simulated asteroid, characterized by the upper limits applied for the outgassing rate and the magnetic moment, is the Mach cone. This structure can be seen in the magnetic field (a) and in the solar wind density $n_{s w}$ (c). It consists of two wings behind the obstacle. One of them shows an enhancement in both the solar wind density and the magnetic field strength. The other a depression in density and in the magnetic field. This Mach cone is formed due to the deflection of the incoming flow. It is also known from the interaction of weak comets with the solar wind (Bagdonat and Motschmann, 2002) and it can be found as well in the MHD simulation of the asteroid Gaspra performed by Baumgärtel et al. (1994). Fig. 8(b) depicts the draping of the magnetic field around the obstacle. It can in addition be seen in this figure that the asteroid triggers a standing wave. This wave is similar to the simulation results presented by Baumgärtel et al. (1997). Hence we assume that this is a whistler wave triggered by the magnetic moment of the asteroid.

The simulation predicts that at a distance of $3000 \mathrm{~km}$ from the asteroid the magnetic field is nearly undisturbed. The magnetic field is only enhanced in the region of the water ion tail. It has a maximum strength of $2.2 \mathrm{nT}$ at this distance.

\subsection{Results}

We did not observe any wave structures in the magnetic field data measured during the flyby. From this and the presented theoretical considerations we infer that the upper limit of the magnetic moment of asteroid (21)Lutetia must be $10^{12} \mathrm{~A} \mathrm{~m}^{2}$.

Despite the remarkably high outgassing (Altwegg et al., this issue) measured by the ROSINA instrument and the resulting structures in the plasma environment, we did not detect any signature in the magnetic field, because the interesting region is much closer to the asteroid than the actual minimum flyby distance of ROSETTA at asteroid (21)Lutetia. Therefore, the lack of a specific signature in the flyby data is not surprising. Furthermore the magnetic measurements neither confirm nor contradict the possible outgassing of water at asteroid (21)Lutetia.

\section{Comparison with other asteroids}

Here we contrast the results obtained at asteroid (21)Lutetia with the characteristics of various other asteroids visited by 

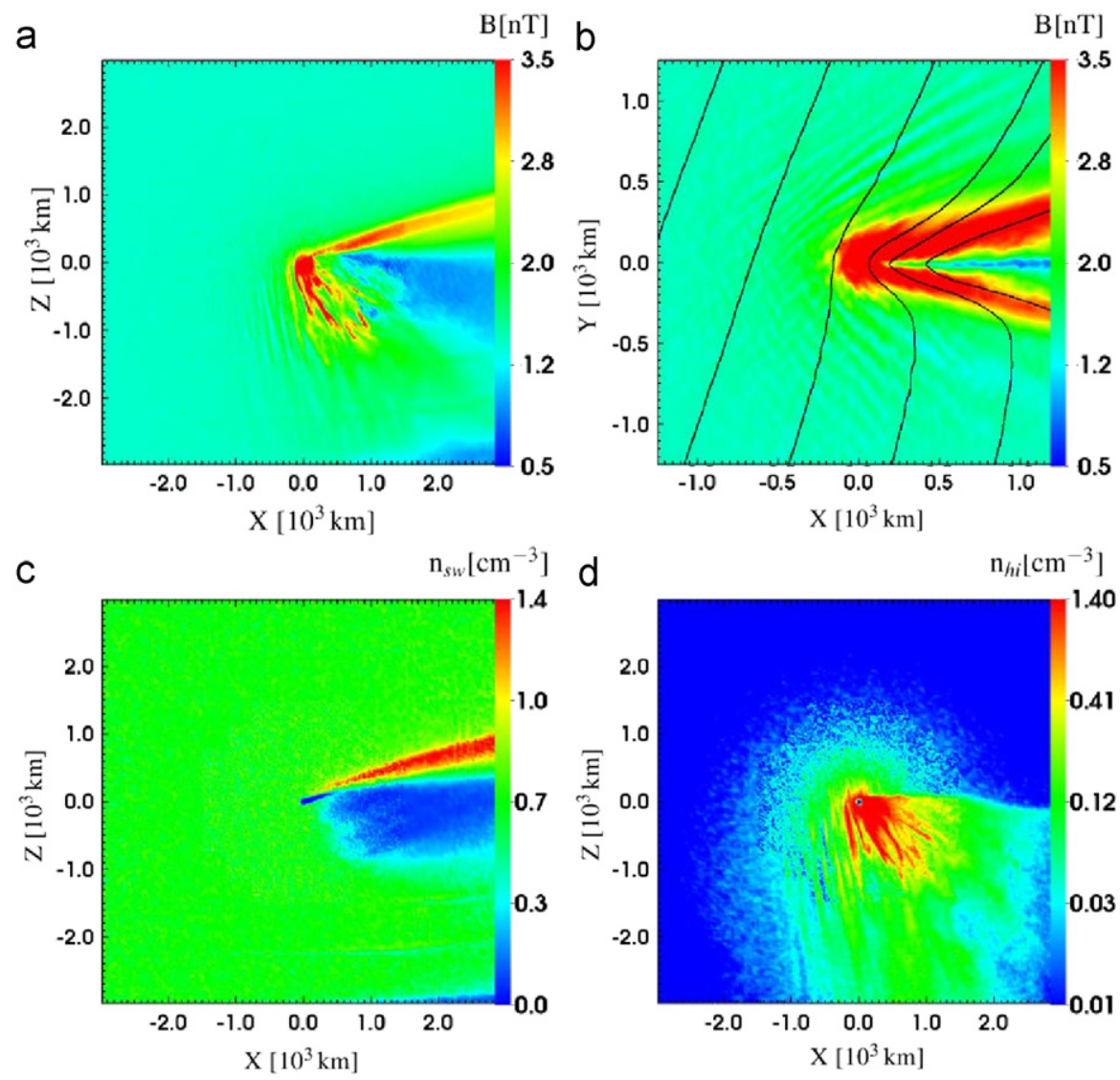

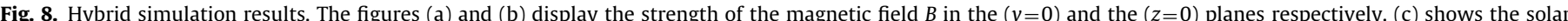

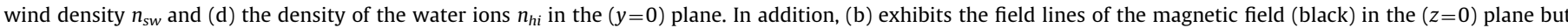

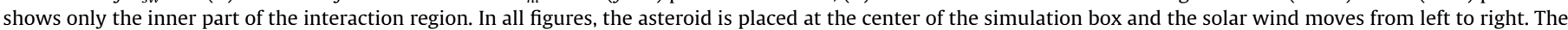

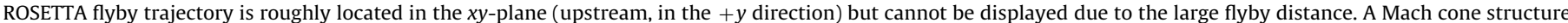

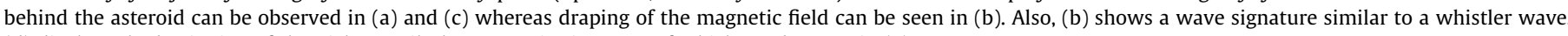
(d) displays the beginning of the pick up tail, the magnetic signature of which can be seen in (a).

Table 1

Selection of relevant asteroidal parameters for the assessment of magnetic properties.

\begin{tabular}{|c|c|c|c|c|c|}
\hline Asteroid & (21)Lutetia & (433)Eros & (951)Gaspra & (2867)Šteins & (9969)Braille \\
\hline Equivalent radius (km) & 49 & 9 & 8 & 3 & 0.8 \\
\hline Dimension $(\mathrm{km})$ & $132 \times 101 \times 93$ & $33 \times 13 \times 13$ & $18.2 \times 10.4 \times 9.4$ & $6.7 \times 5.8 \times 4.5$ & $2,2 \times 1 \times 1$ \\
\hline Volume $\left(\mathrm{km}^{3}\right)$ & 500000 & 2500 & 2145 & 113 & 2 \\
\hline Density $\left(\mathrm{kg} / \mathrm{m}^{3}\right)$ & 3400 & 2650 & 3900 & 3200 & 3900 \\
\hline Material & Carb. Chon., Metal & Chondrites & Metal, Oliv., Pyrox. & Enstatite & Pyroxene, Olivine \\
\hline Spectral class & $\mathrm{X}_{\mathrm{c}}$-type & S-type & S-type & E-type & Q-type \\
\hline Mass (kg) & $1.70 \times 10^{18}$ & $6.63 \times 10^{15}$ & $8.36 \times 10^{15}$ & $3.62 \times 10^{14}$ & $7.75 \times 10^{12}$ \\
\hline Encounter & ROSETTA 10.7 .2010 & NEAR: 12.2.2001 & Galileo: 29.10.1991 & ROSETTA: 5.9 .2008 & DS1: 29.07.1999 \\
\hline Solar distance (AU) & 2.72 & 1.75 & 2.2 & 2.14 & 1.32 \\
\hline Encounter distance $R(\mathrm{~km})$ & 3120 & Landing & 1600 & 800 & 28 \\
\hline Magnetic field (nT) @ $R$ & $<1$ & $<5$ & DRAPING & $<1$ & 2 \\
\hline Max. dipole moment $\left(\mathrm{A} \mathrm{m}^{2}\right)$ & $1.0 \times 10^{12}$ & $1.3 \times 10^{10}$ & $2.0 \times 10^{14}$ & $1.0 \times 10^{12}$ & $2.2 \times 10^{11}$ \\
\hline Max. magnetization $(\mathrm{A} / \mathrm{m})$ & $2.0 \times 10^{-3}$ & $5.0 \times 10^{-3}$ & 93 & 9 & 110 \\
\hline Spec. moment $\left(\mathrm{A} \mathrm{m}^{2} / \mathrm{kg}\right)$ & $5.9 \times 10^{-7}$ & $1.9 \times 10^{-6}$ & $2.4 \times 10^{-2}$ & $2.8 \times 10^{-3}$ & $2.8 \times 10^{-2}$ \\
\hline
\end{tabular}

spacecraft in the past. An overview of relevant asteroidal parameters in terms of magnetic investigations is given in Table 1. The values found during previous encounters were compiled using the data and results listed, inter alia, in Weiss et al. (this issue), Acuña et al. (2002), Kivelson et al. (1993), Auster et al. (2010), Accomazzo et al. (2010), and Richter et al. (2001). The table is ordered by the size of the celestial bodies. With its $\sim 49 \mathrm{~km}$ radius (21)Lutetia is by far the biggest asteroid, whereas (9969)Braille with its $0.8 \mathrm{~km}$ radius constitutes a tiny example. The density of all bodies varies in the range of $2650-3900 \mathrm{~kg} / \mathrm{m}^{3}$, while (433)Eros is the most porous of these bodies. The related masses of the asteroids decrease by about six orders of magnitude from (21)Lutetia to (9969)Braille. The characterization of the magnetic properties is given by the upper limit of the global magnetization. The lowest values were derived from the ROSETTA flyby of asteroid (21)Lutetia and the landing of the NEAR spacecraft on asteroid (433)Eros. Both values are of the order of $10^{-3} \mathrm{~A} / \mathrm{m}$. On the other hand the largest values of $\sim 100 \mathrm{~A} / \mathrm{m}$ (i.e. about five orders of magnitude higher than the values previously mentioned) were determined during the 
GALILEO flyby of asteroid (951)Gaspra and the DEEP SPACE 1 encounter with asteroid (9969)Braille. The ROSETTA flyby at asteroid (2867)Šteins constrained medium global magnetization to a value of $\sim 10 \mathrm{~A} / \mathrm{m}$.

Magnetic characterization was derived in different ways at different asteroids. In the case of asteroid (9969)Braille, Richter et al. (2001) made a direct measurement of the magnetic field ( $2 \mathrm{nT}$ at a $28 \mathrm{~km} \mathrm{C/A} \mathrm{distance)} \mathrm{which} \mathrm{was} \mathrm{fitted} \mathrm{by} \mathrm{a} \mathrm{centered}$ dipole. At asteroid (433)Eros the NEAR spacecraft not only performed a flyby but also a landing and accordingly Acuña et al. (2002) made a direct magnetic field measurement that provided an absolute value of less than $5 \mathrm{nT}$ (including uncertainties) at the landing site. In contrast to these activities Kivelson et al. (1993) measured field draping at asteroid (451)Gaspra and derived the magnetic dipole moment from the required standoff distance for the assumed magnetosphere (Baumgärtel et al., 1994). However, Blanco-Cano et al. (2003) suggested, based on their own simulations, that the observed draping might have been also due to the effect of a rotating solar wind structure rather than due to the asteroid. During the flybys at asteroids (2867)Šteins and (21)Lutetia the ROSETTA magnetometers did not detect any evidence of draping nor did they measure a prominent signature of more than $1 \mathrm{nT}$. Thus the upper limits for the magnetic properties of these asteroids have been estimated using the theoretical considerations of Baumgärtel et al. (1997) and Simon et al. (2006).

\section{Summary and conclusions}

At the conclusion of the present investigations, it can be stated that despite of the magnetic interference generated by the ROSETTA spacecraft, interesting magnetic field features at asteroid (21)Lutetia were identified. From the magnetic point of view a much closer flyby at asteroid (21)Lutetia would have been preferable but this was not feasible due to reasons of spacecraft safety and camera field-of-view constraints.

All three magnetometer sensors onboard ROSETTA were operating during the flyby and they obtained high resolution time series of magnetic field data. Due to spacecraft disturbances these data are highly disturbed, especially the data of the Lander magnetometer ROMAP. Nevertheless these measurements could be used in conjunction with theoretical studies, to derive upper limits for the global magnetic properties of asteroid (21)Lutetia. The maximal dipole moment could be set at $1.0 \times 10^{12} \mathrm{~A} \mathrm{~m}^{2}$, the maximal global magnetization at $2.1 \times 10^{-3} \mathrm{~A} / \mathrm{m}$, and the specific moment to $5.9 \times 10^{-7} \mathrm{~A} \mathrm{~m}^{2} / \mathrm{kg}$. Due to the huge flyby distance it is only possible to provide estimates of these global magnetic properties.

Draping of the magnetic field around the nucleus was sought, but evidence for this could not be confirmed as the signal to noise ratio (due to the large distance concerned and the spacecraft generated disturbances) was too low. The results obtained at Lutetia were compared with the results of flybys made at different asteroids visited during recent space missions. Lutetia is the largest asteroid considered.

Plasma simulations taking into account possible outgassing and magnetization, predicted the presence of interesting structures close to the asteroid but these would not have been detected at the large flyby distance of $3120 \mathrm{~km}$.

\section{Acknowledgments}

The work of H.U. Auster, K.-H. Glassmeier, C. Koenders, and I. Richter was financially supported by the German Bundesministerium für Wirtschaft und Technologie and the Deutsches
Zentrum für Luft- und Raumfahrt under contract 50 QP 1001 for ROSETTA. The plasma simulation was performed on the system of the North-German Supercomputing Alliance. All computations concerning the $\mathrm{s} / \mathrm{c}$ position and orientation have been calculated with use of the SPICE software developed by NASA's NAIF team. Special thanks go to Boris Semenov from the NAIF team, who provided the needed GASPRA kernel in a quick and unbureaucratic way. Operation of the RPC instrumentation is supported at Imperial College London by the UK Science and Technology Facilities Council. We highly appreciate the support of C.D. Fry (Exploration Physics International Inc, Huntsville), who estimated the IMF conditions for the flyby time using the HAFV. 2 model. Last but not least we thank the ROSETTA mission operations team at ESOC who did an excellent job and provided for a smooth flyby.

\section{References}

Acton, C.H., 1996. Ancillary data services of NASA's navigation and ancillary information facility. Planetary and Space Science 44, 65-70.

Accomazzo, A., Wirth, K.R., Lodiot, S., Küppers, M., Schwehm, G., 2010. The flyby of ROSETTA at asteroid Šteins-mission and science operations. Planetary and Space Science 58, 1058-1065.

Acuña, M.H., Wasilewski, P., Kletetshka, G., Russell, C.T., Anderson, B.J., Zanetti, L., Omidi, N., 2002. NEAR magnetic field observations at 433 Eros: first measurements from the surface of an asteroid. Icarus 155, 220-228.

Altwegg, K., Balsiger, H., Calmonte, U., Hässig, M., Hofer, L., Jäckel, A., Schläppi, B., Wurz, P., Berthelier, J.J., De Keyser, J., Fiethe, B., Fuselier, S., Mall, U., Rème, H., Rubin, M. In situ mass spectrometry during the Lutetia flyby. Planetary and Space Science, this issue. doi:10.1016/j.pss.2011.08.011.

Anderson, B.J., Zanetti, L.J., Lohr, D.H., Hayes, J.R., Acuña, M.H., Russell, C.T., Mulligan, T., 2001. In-Flight Calibration of the NEAR magnetometer. IEEE Transactions on Geoscience and Remote Sensing 39, 907-917.

Arge, C.N., Pizzo, V., 2000. Improvement in the prediction of solar wind conditions using near-real time solar magnetic field updates. Journal of Geophysical Research 105, 10465-10480.

Auster, H.U., Apathy, I., Berghofer, G., Remizov, A., Roll, R., Fornaçon, K.H., Glassmeier, K.-H., Haerendel, G., Hejja, I., Kührt, E., Magnes, W., Moehlmann, D., Motschmann, U., Richter, I., Rosenbauer, H., Russell, C.T., Rustenbach, J., Sauer, K., Schwingenschuh, K., Szemerey, I., Waesch, R., 2007. ROMAP: ROSETTA magnetometer and plasma monitor. Space Science Reviews 128.

Auster, H.U., Richter, I., Glassmeier, K.-H., Berghofer, G., Carr, C.M., Motschmann, U., 2010. Magnetic field investigations during ROSETTA's 2867 Šteins flyby. Planetary and Space Science 58, 1124-1128.

Bagdonat, T.B., Motschmann, U., 2002. From a weak to a strong comet-3D Global hybrid simulation studies. Earth, Moon and Planets 90, 305-321.

Balsiger, H., Altwegg, K., Bochsler, P., Eberhardt, P., Fischer, J., Graf, S., Jäckel, A. Kopp, E., Langer, U., Mildner, M., Müller, J., Riesen, T., Rubin, M., Scherer, S. Wurz, P., Wüthrich, S., Arijs, E., Delanoye, S., de Kayser, J., Neefs, E., Nevejans, D., Réme, H., Aoustin, C., Mazelle, C., Bertaux, J.-L., Duvet, L., Illiano, J.-M., Fuselier, S.A., Ghielmetti, A.G., Magoncelli, T., Shelley, E.G., Korth, A., Heerlein, K., Lauche, H., Livi, S., Loose, A., Mall, U., Carignan, G.R., Fisk, L.A., Waite, J.H., Young, D.T., Wollnik, H., 2007. ROSINA-ROSETTA orbiter spectrometer for ion and neutral analysis. Space Science Reviews 128, 745-801. doi:10.1007/ s11214-006-8335-3.

Barucci, M.A., Fulchignoni, M., Rossi, A., 2007. ROSETTA asteroid targets: 2867 Steins and 21 Lutetia. Space Science Reviews 128, 67-78. doi:10.1007/s11214006-9029-6.

Baumgärtel, K., Sauer, K., Bogdanov, A., 1994. A magnetospheric model of solar wind interaction with asteroid Gaspra. Science 263, 653-655.

Baumgärtel, K., Sauer, K., Story, T.R., 1997. Solar wind response to a magnetized asteroid: linear theory. Icarus 129, 94-105.

Blanco-Cano, X., Omidi, N., Russell, C.T., 2003. Hybrid simulations of solar wind interaction with magnetized asteroids: comparison with Galileo observations near Gaspra and Ida. Journal of Geophysical Research 108, A5, 1216, doi: 10. 1029/2002JA009618.

Boesswetter, A., Auster, U., Richter, I., Fränz, M., Langlais, B., McKenna-Lawlor, S., Simon, S., Motschmann, U., Glassmeier, K.-H., Edberg, N.J.T., Lundin, R., 2009. Rosetta swing-by at Mars - an analysis of the ROMAP measurements in comparison with results of 3-D multi-ion hybrid simulations and MEX/ ASPERA-3 data. Annales Geophysicae 27, 2383-2398.

Burch, J.L., Goldstein, R., Cravens, T.E., Gibson, W.C., Lundin, R.N., Pollock, C.J., Winningham, J.D., Young, D.T., 2007. RPC-IES: the ion and electron sensor of the ROSETTA plasma consortium. Space Science Reviews 128, 697-712. doi:10.1007/s11214-006-9002-4.

Carr, C.M., Cupido, E., Lee, C.G.Y., Balogh, A., Beek, T., Burch, J.L., Dunford, C.N., Eriksson, A.I., Gill, R., Glassmeier, K.-H., Goldstein, R., Lagoutte, D., Lundin, R., Lundin, K., Lybekk, B., Michau, J.L., Musmann, G., Nilsson, H., Pollock, C., Richter, I., Trotignon, J.G., 2007. RPC: the Rosetta plasma consortium. Space Science Reviews 128, 629-647. 
Eastwood, J.P., Schwartz, S.J., Horbury, T.S., Carr, C.M., Glassmeier, K.-H., Richter, I., Koenders, C., Plaschke, F., Wild, J.A., 2011. Transient Pc3 wave activity generated by a hot flow anomaly: Cluster, Rosetta, and ground based observations. Journal of Geophysical Research 116, A08224. doi:10.1029/ 2011JA016467.

Edberg, N.J.T., Eriksson, A.I., Auster, U., Barabash, S., Bößwetter, A., Carr, C.M., Cowley, S.W.H., Cupido, E., Fränz, M., Glassmeier, K.-H., Goldstein, R., Lester, M., Lundin, R., Modolo, R., Nilsson, H., Richter, I., Samara, M., Trotignon, J.G., 2008. Simultaneous measurements of Martian plasma boundaries by Rosetta and Mars Express. Planetary and Space Science 57, 1085-1096.

Edberg, N.J.T., Auster, U., Barabash, S., Bößwetter, A., Brain, D.A., Burch, J.L., Carr, C.M., Cowley, S.W.H., Cupido, E., Duru, F., Eriksson, A.I., Fränz, M., Glassmeier, K.-H., Goldstein, R., Lester, M., Lundin, R., Modolo, R., Nilsson, H., Richter, I., Samara, M., Trotignon, J.G., 2009. Rosetta and Mars Express observations of the influence of high solar wind pressure on the Martian plasma environment. Annales Geophysicae 27, 4533-4545.

Eriksson, A.I., Boström, R., Gill, R., Åhlén, L., Jansson, S.-E., Wahlund, J.-E., André, M., Mälkki, A., Holtet, J.A., Lybekk, B., Pedersen, A., Blomberg, L.G., 2007. The LAP Team, RPC-LAP: the ROSETTA Langmuir probe instrument. Space Science Reviews 128, 729-744. doi:10.1007/s11214-006-9003-3.

Fry, C.D., Dryer, M., Smith, Z., Sun, W., Deehr, C.S., Akaosfu, S.-I., Smith, Z., 2003. Forecasting solar wind structures and shock arrival times using an ensemble of models. Journal of Geophysical Research 108, 1070. doi:10.1029/ 2002JA009474.

Goesmann, F., Rosenbauer, H., Roll, R., Szopa, C., Raulin, F., Sternberg, R., Israel, G., Meierhenrich, U., Thiemann, W., Munoz-Caro, G., 2007. Cosac, the cometary sampling and composition experiment on philae. Space Science Reviews 128, 257-280.

Glassmeier, K.-H., Boehnhardt, H., Koschny, D., Kührt, E., Richter, I., 2007a. The ROSETTA Mission: flying towards the origin of the solar system. Space Science Reviews 128, 1-21.

Glassmeier, K.-H., Richter, I., Diedrich, A., Musmann, G., Auster, U., Motschmann, U., Balogh, A., Carr, C., Cupido, E., Coates, A., Rother, M., Schwingenschuh, K., Szegö, K., Tsurutani, B., 2007b. RPC-MAG: the Fluxgate Magnetometer in the ROSETTA Plasma Consortium. Space Science Reviews 128, 649-670.

Greenstadt, E., 1971. Conditions for magnetic interaction of asteroids with the solar wind. Icarus $14,374-381$.

Hansen, K.C., Bagdonat, T., Motschmann, U., Alexander, C., Combi, M.R., Cravens, T.E., Gombosi, T.I., Jia, Y.-D., Robertson, I.P., 2007. The plasma environment of Comet 67P/Churyumov-Gerasimenko throughout the ROSETTA main mission. Space Science Reviews 128, 133-166.

Hedgecock, P.C., 1975. A correlation technique for magnetometer zero level determination. Space Science Instrumentation 1, 83-90.

Ip, W.-H., Herbert, F., 1982. On the asteroidal conductivities as inferred from meteorites. The Moon and Planets 28, 43-47.

Kivelson, M.G., Bargatze, L.F., Khurana, K.K., Southwood, D.J., Walker, R.J., Coleman, P.J., 1993. Magnetic field signatures near Galileo's closest approach to Gaspra. Science 261, 331-334.

Kivelson, M.G., Khurana, K.K., Means, J.D., Russell, C.T., Snare, R.C., 1995. Galileo Orbiter Magnetic Field Calibrated Data Record, Gaspra Flyby. NASA Planetary Data System, GO-A-MAG-3-RDR-GASPRA-HIGH-RES-V1.0, vol. GOMW 0004.

Leinweber, H.K., Russell, C.T., Torkar, K., Zhang, T.L., Angelopoulos, V., 2008. An advanced approach to finding magnetometer zero levels in the interplanetary field. Measurement Science and Technology 19.
McKenna-Lawlor, S.M.P., Dryer, M., Fry, C.D., Smith, Z.K., Intriligator, D.S., Courtney, W.R., Deehr, C.S., Sun, W., Kecskemety, K., Kudela, K., Balaz, J., Barabash, S. Futaana, Y., Yamauchi, M., Lundin, R., 2008. Predicting interplanetary shock arrivals at Earth, Mars and Venus: a real-time modeling experiment following the solar flares of 5-14 December, 2006. Journal of Geophysical Research 113, A06101. doi:10.1029/2007JA012577.

Müller, J., Simon, S., Motschmann, U., Schüle, J., Glassmeier, K.-H., Pringle, G., 2011 A.I.K.E.F.: adaptive hybrid model for space plasma simulations. Computer Physics Communications 182, 946-966.

Musmann, G., Neubauer, F.M., Lammers, E., 1977. Radial variation of the interplanetary magnetic field between 0.3 and $1.0 \mathrm{AU}$ : observations by the HELIOS 1 Spacecraft. Journal of Geophysical Research 42, 591-598.

Ness, N.F., 1971. Interaction of the solar wind with the moon. Physics of the Earth and Planetary Interiors 4, 197-198.

Nilsson, H., Lundin, R., Lundin, K., Barabash, S., Borg, H., Norberg, O., Fedorov, A. Sauvaud, J.-A., Koskinen, H., Kallio, E., et al., 2007. RPC-ICA: the ion composition analyzer of the Rosetta plasma consortium. Space Science Reviews 128 671-695. doi:10.1007/s11214-006-9031-z.

Richter, I., Brinza, D.E., Cassel, C., Glassmeier, K.-H., Kuhnke, F., Musmann, G., Othmer, C., Schwingenschuh, K., Tsurutani, B., 2001. First direct magnetic field measurements of an asteroidal magnetic field: DS1 at Braille. Geophysical Research Letters 28, 1913-1916.

Richter, I., Koenders, C., Glassmeier, K.-H., Tsurutani, B.T., Goldstein, R., 2011. Deep Space 1 at comet 19P/Borrelly: magnetic field and plasma observations. Planetary and Space Science. doi:10.1016/j.pss.2011.02.001.

Runcorn, S.K., 1975a. An ancient lunar magnetic dipole field. Nature 253, 701-703. doi:10.1038/253701a0.

Runcorn, S.K., 1975b. On the interpretation of lunar magnetism. Physics of the Earth and Planetary Interiors 10, 327-335.

Schwehm, G., Schulz, R., 1999. ROSETTA goes to Comet Wirtanen. Space Science Reviews 90, 313-319.

Simon, S., Bagdonat, T., Motschmann, U., Glassmeier, K.-H., 2006. Plasma environment of magnetized asteroids: a 3-D hybrid simulation study. Annales Geophysicae 24, 407-414.

Trotignon, J.G., Michau, J.L., Lagoutte, D., Chabassière, M., Chalumeau, G., Colin, F. Décréau, P.M.E., Geiswiller, J., Gille, P., Grard, R., Hachem, T., Hamelin, M., Eriksson, A., Laakso, H., Lebreton, J.P., Mazelle, C., Randriamboarison, O. Schmidt, W., Smit, A., Telljohann, U., Zamora, P., 2007. RPC-MIP: the mutua impedance probe of the Rosetta plasma consortium. Space Science Reviews 128, 713-728. doi:10.1007/s11214-006-9005-1.

Wallis, M.K., 1986. Bow shock decay in comets Giacobini-Zinner and Halley. Advances in Space Research 6, 239-242.

Wang, Y.M., Sheely, N.R., 1990. Solar wind speed and coronal flux tube expansion The Astrophysical Journal 355, 726-732.

Weiss, B.P., Elkins-Tanton, L.T., Stanley, S., Lima, E.A., Carporzen, L., 2008. Science 322, 713-716.

Weiss, B.P., Gattacceca, J., Stanley, S., Rochette, P., Christensen, U.R., 2010. Space Science Reviews 152, 341-390.

Wright, I.P., Barber, S.J., Morgan, G.H., Morse, A.D., Sheridan, S., Andrews, D.J. Maynard, J., Yau, D., Evans, S.T., Leese, M.R., Zarnecki, J.C., Kent, B.J., Waltham, N.R., Whalley, M.S., Heys, S., Drummond, D.L., Edeson, R.L., Sawyer, E.C., Turner, R.F., Pillinger, C.T., 2007. PTOLEMY-An instrument to measure stable isotopic ratios of key volatiles on a cometary nucleus. Space Science Reviews 128, 363-381. doi:10.1007/s11214-006-9001-5. 